\title{
Rapid-Deployment Aortic Valve Replacement for a Hemodialysis Patient with Prior Coronary Artery Bypass Grafting
}

\author{
Taira Yamamoto, PhD, ${ }^{1}$ Daisuke Endo, $\mathrm{PhD},{ }^{1}$ Hironobu Yamaoka, $\mathrm{PhD},{ }^{2}$ Akie Shimada, $\mathrm{PhD},{ }^{1}$ \\ Satoshi Matsushita, $\mathrm{PhD},{ }^{1}$ Atsushi Amano, $\mathrm{PhD}^{1}$ \\ ${ }^{1}$ Department of Cardiovascular Surgery, Juntendo University, Tokyo, Japan; \\ ${ }^{2}$ Department of Cardiovascular Surgery, Edogawa Hospital, Tokyo, Japan
}

\section{ABSTRACT}

Background: Aortic valve reoperation increases the risk of mortality and morbidity. The 2017 European Society of Cardiology guidelines for managing valvular heart disease with a previous heart surgery and intact bypass grafts consider patients with high surgical risk to be injury-prone during sternotomy. In high-risk patients with prior coronary artery bypass grafting, several authors have reported the noninferiority or superiority of transcatheter aortic valve replacement (TAVR) compared with surgical aortic valve replacement; however, in Japan, TAVR cannot be performed for patients on hemodialysis. In this study, we report a case of successful implantation of the new rapid-deployment bioprosthesis in a 65-year-old Japanese man on dialysis with prior coronary artery bypass grafting.

Methods: The rapid-deployment aortic valve system has demonstrated excellent hemodynamic performance, durability, and safety. However, implantation requires specific training and the analysis of preoperative 3D computed tomographic imaging. The cineangiography revealed patency of all grafts, and the saphenous vein graft (SVG) had overlapped the planned aortotomy position. By avoiding the anastomotic part of the SVG, we could perform rapid-deployment aortic valve replacement efficiently even if the aortic incision was repositioned, and the incision was smaller than planned.

Results: We used the $23-\mathrm{mm}$ Intuity valve without an additional stitch, and the cardiopulmonary bypass and aortic cross-clamp times were only 52 and 39 minutes, respectively.

Conclusion: This novel valve may be beneficial in complex combinational procedures for hemodialysis patients with prior coronary artery bypass grafting.

Received December 14, 2020; accepted Fanuary 13, 2021.

Correspondence: Taira Yamamoto, Department of Cardiovascular Surgery, Funtendo University, 2-1-1, Bukyo-ku, Tokyo, Fapan; 03-3813-3210 (e-mail: tyamamo@juntendo.ac.jp).

\section{INTRODUCTION}

Aortic valve reoperation increases the risk of mortality and morbidity. The 2017 European Society of Cardiology guidelines for managing valvular heart disease with previous heart surgery and intact bypass grafts consider patients with high surgical risk to be injury-prone during sternotomy. The cardiology team is recommended to consider transcatheter aortic valve replacement (TAVR) or surgical aortic valve replacement (SAVR) in patients with high surgical risk [Conte 2016].

In high-risk patients with prior coronary artery bypass grafting (CABG), the noninferiority or superiority of TAVR compared with SAVR has been reported in a subgroup analysis of randomized trials, retrospective single-center studies, and meta-analyses [Coti 2020; Ando 2016; Kocher 2013; Baumgartner 2017].

In Japan, however, TAVR cannot be performed for patients on hemodialysis. Recently, SAVR using rapid-deployment valves (Intuity Aortic Valve system; Edwards Life Sciences, Irvine, CA) has been introduced as an effective method for high-risk patients. Rapid-deployment aortic valve replacement (RD-AVR) shortens the duration aortic cross-clamp time, cardiopulmonary bypass (CPB) time, and hospital stay [Jegaden 2012]. Herein, we report a case of RD-AVR in a hemodialysis patient with prior CABG and explain the situations in which this system is incredibly useful. Additionally, we offer insights on the use of preoperative diagnostic imaging to predict the suitability of this system in a patient.

\section{CASE REPORT}

A 65-year-old man presented with complaints of exertional dyspnea. He experienced palpitations when climbing the stairs 2 months prior, and his dyspnea had worsened. Seven years before, a CABG (left internal thoracic artery to left anterior descending artery [LITA-LAD] and saphenous vein graft to posterior descending artery [SVG-PD]) was performed on the beating heart without $\mathrm{CPB}$. He had immunoglobulin A nephropathy at the age of 33 years and chronic renal failure at 44. He had a medical history of hyperlipidemia (low-density lipoprotein cholesterol $100 \mathrm{mg} / \mathrm{dl}$ ), and was on continuous statin therapy postoperatively. He had no history of smoking.

Physical examination revealed a height and weight of 152.5 $\mathrm{cm}$ and $45.9 \mathrm{~kg}$, respectively. His blood pressure was 130/56 
$\mathrm{mmHg}$, and he had a heart rate of $77 \mathrm{bpm}$, in sinus rhythm. His oral medications were atorvastatin calcium hydrate 10 $\mathrm{mg} /$ day, lanthanum carbonate hydrate $1500 \mathrm{mg} /$ day, ferric citrate hydrate $250 \mathrm{mg}$ /day, cinacalcet hydrochloride $50 \mathrm{mg}$ / day, and precipitated calcium carbonate $1500 \mathrm{mg} /$ day.

A normal sinus rhythm was revealed on electrocardiogram (ECG). The diameter of the ascending aorta was $38 \mathrm{~mm}$ on computed tomography (CT). Echocardiography revealed severe aortic valve stenosis. The aortic valve area was $0.8 \mathrm{~cm} 2$, velocity was $4.0 \mathrm{~m} / \mathrm{s}$, and the mean pressure gradient was 40 $\mathrm{mmHg}$. His left ventricular ejection fraction was $68 \%$. Cineangiography revealed patency of all grafts, $100 \%$ occlusion in the midportion of the right coronary artery, $90 \%$ stenosis in the proximal left anterior descending artery, and 90\% stenosis in the high lateral branch (Figures 1A to 1D). Initially, we strategized to perform primary percutaneous coronary intervention (PCI) in the high lateral branch with a stent (Ultimaster $2.25 \times 18 \mathrm{~mm}$; Termo Corporation, Tokyo, Japan)

Japan's medical insurance system does not cover TAVR; therefore, a conventional SAVR was scheduled 1 month after
PCI (risk score: Society of Thoracic Surgeons score $7.81 \%$, EuroSCORE II 7.15\%, Japan Score 9.9\%). An ECG-gated cardiac 3D CT was performed preoperatively, and the positions of each commissure and nadir were found to be on the same level in this case. The left ventricular outflow tract (LVOT) morphology was not circular; however, calcification was observed. Using preoperative imaging, a $23-\mathrm{mm}$ prosthetic valve was predicted to be appropriate for the artificial valve. However, the SVG overlapped the location of the aortic incision planned using a preoperative CT scan (Figure 2 ). Because this was a high-risk case, we chose the RD-AVR system with a short operative time that can be used even for a small aortotomy to avoid proximal anastomoses of the SVG-PD.

A sternotomy was performed similar to the previous surgery. After adhesion detachment, an artificial CPB was established with the inflow on the ascending aorta, the outflow on the superior vena cava, and the right femoral vein. We exfoliated the tissue surrounding the SVG and placed a tape around the SVG. The calcifications of both the aortic valves,
A

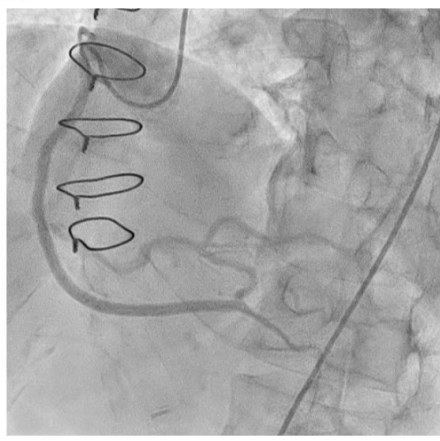

B

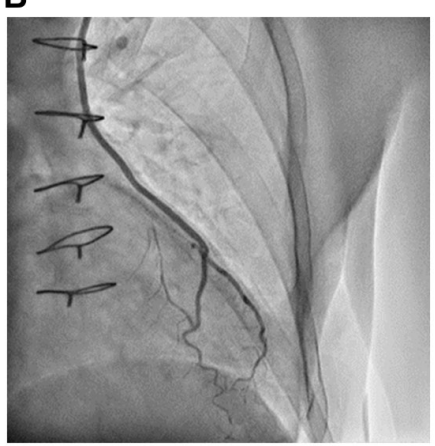

C

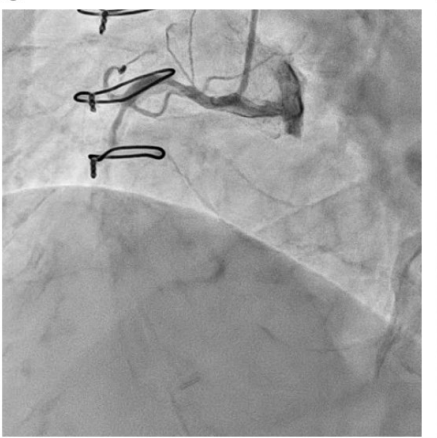

D

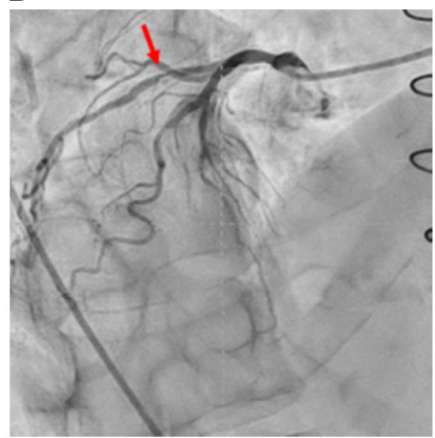

Figure 1. CABG. A, SVG-4PD was patent. B, LITA-D-LAD was patent. C, The proximal right coronary artery was occluded. D, The proximal left anterior descending artery was occluded, and $75 \%$ stenosis was observed at the obtuse marginal branch (red arrow). LITA-D-LAD indicates left internal thoracic artery to left anterior descending artery; SVG-4PD, saphenous vein graft to the posterior descending artery.

A

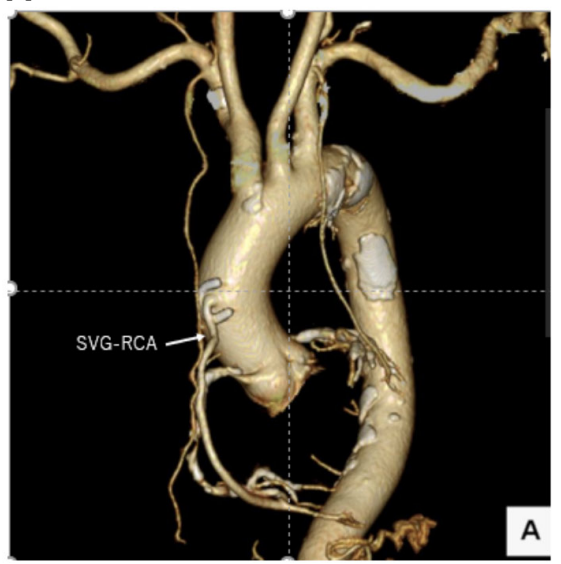

B

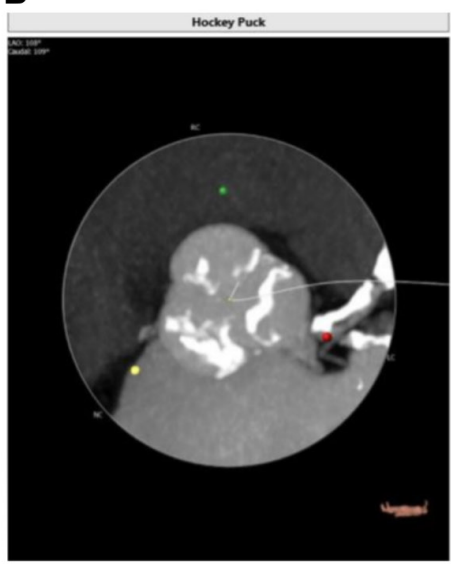

C

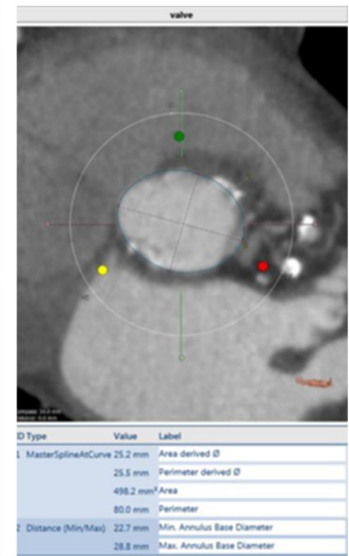

D

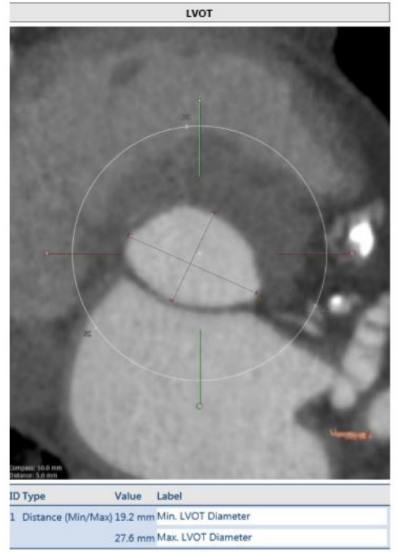

Figure 2. ECG-gated 3D CT. A, White arrow is the SVG-PD. SVG overlapped the location of the aortic incision planned. B, Calcification at the aortic valve. C, The morphology of the annulus was almost a circle; the annulus is a circle, $22.8 \times 25.2 \mathrm{~mm}$. D, The LVOT was oval. No calcification in the LVOT. 
the tricuspid and annulus, were severe. After excising the valve and calcification, 3 fixed needles were placed in each nadir, and the artificial valve was set using the $23-\mathrm{mm}$ Intuity valve system. This prosthetic valve was of the size predicted by the preoperative 3D CT, with no gaps or additional stitch (Figures $3 \mathrm{~A}$ and $3 \mathrm{~B}$ ).

The operative, $\mathrm{CPB}$, and aortic cross-clamp times were 201, 52, and 39 minutes, respectively. The respiratory support time was 5 hours postoperatively. The patient was discharged from the intensive care unit on the second postoperative day, and rehabilitation was initiated. No postoperative atrial fibrillation, complete atrioventricular block, or other complications occurred. One year after the operation, the patient had resumed his day-to-day activities effectively. The hemodynamic performance in the prosthetic valve has been excellent throughout, without valvular regurgitation. Doppler ultrasonography (12 months after surgery) revealed an effective orifice area (EOA) of $2.2 \mathrm{~cm}^{2}, \mathrm{EOA}$ index of 1.57

\section{A}

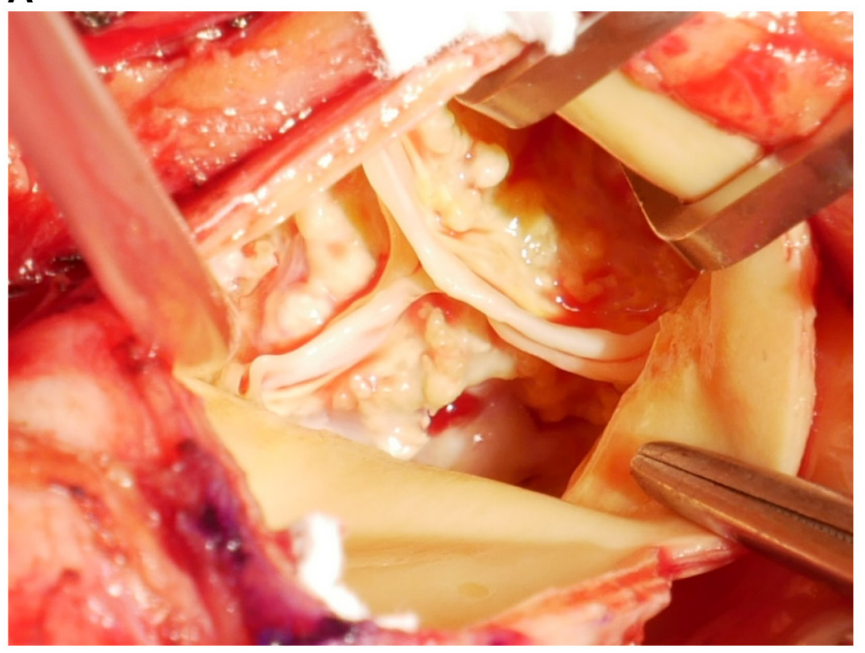

B

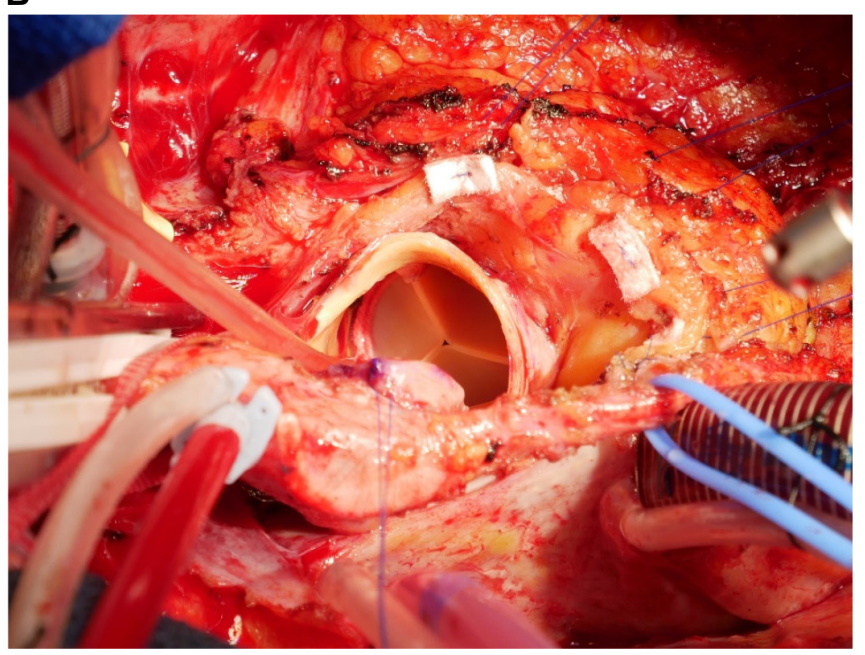

Figure 3. Operative findings. A, Calcified tricuspid aortic valve. B, The RD-AV system was replaced. A blue tape was put around the SVG. $\mathrm{cm}^{2} / \mathrm{m}^{2}$, peak jet velocity of $1.8 \mathrm{~m} / \mathrm{s}$, peak pressure gradient of $7 \mathrm{mmHg}$, and left ventricular ejection fraction of $74 \%$.

\section{DISCUSSION}

TAVR has been widely accepted as the standard approach in high-risk elderly patients. However, TAVR may contribute to increased incidence of periprosthetic valve regurgitation, the prevalence of conduction disorders, and the risk of subclinical leaflet thrombosis [Ando 2016]. Therefore, given the technical complications with current TAVR, SAVR is still necessary.

Aortic valve surgery has significantly advanced in recent years with the introduction of minimally invasive procedures and new devices. Surgical rapid-deployment aortic bioprostheses have been developed as new prosthetic valve sewing methods, and an excellent 1-year postsurgery outcome has been reported [Andreas 2019]. One such surgical device is the RD-AVR system. Andreas et al [2019] reported that the intermediate-term outcome of $500 \mathrm{RD}$-AVR systems demonstrated excellent hemodynamic performance, durability, and safety [Andreas 2019].

However, Andreas et al [2019] also reported concerns regarding perivalvular leakage and the new implantation rate of pacemakers [Andreas 2019]. A complete atrioventricular block can be avoided by making the artificial valve sewing part $1 \mathrm{~mm}$ higher. The incidence of perivalvular regurgitation has been reported as $2 \%$ to $4 \%$ previously [Jegaden 2012; Berretta 2020]. The regurgitation occurs mostly between the right coronary cusp-noncoronary cusp commissure and the noncoronary cusp nadir. Andreas et al [2019] reported adding an extra stitch in the noncoronary sinus in selected patients [Andreas 2019]. We added an extra stitch in the commissure between the right coronary cusp and noncoronary cusp.

Specific training for RD-AVR implantation, preoperative analysis using ECG-gated 3D CT, and appropriate artificial valve size selection are critical. In this case, we predicted that we could use a $23-\mathrm{mm}$ prosthetic valve from preoperative ECG-gated 3D CT measurements. Moreover, the commissure position and annulus and LVOT morphologies helped in concluding that no extra stitch would be required, since no gaps between the native annulus and artificial valve were created. We used the 23-mm Intuity valve without an additional stitch, and the $\mathrm{CPB}$ and aortic cross-clamp times were only 52 and 39 minutes, respectively.

Regarding reoperation, the difficulty of a procedure increases if the previous bypass graft is patent. Particularly, if the SVG-4PD is patent and the running of the graft interferes with the surgical operation, it may be necessary to remove the bypass graft once and revascularize it. To avoid the anastomotic part of the SVG, even if the position of the aortotomy has changed and the incision is small, RD-AVR can be performed easily.

Patients on dialysis have a limited lifespan, but mechanical valves are superior to biological valves for midterm results [Greason 2014]. Rehospitalization due to bleeding was a little more frequent with mechanical valves, but Chan et al 
[2019] reported that the use of mechanical valves in patients on dialysis is appropriate Chan et al [2019] compared 323 patients with a biological valve and 168 patients with a mechanical valve and reported no significant differences in in-hospital mortality, 5-year postoperative survival, or 5-year reoperation-free rate Nakatsu et al [2019]. We believe that multiple factors should be considered when choosing a valve for patients with aortic valve stenosis on hemodialysis. For example, one should prioritize AVR with bioprosthetic valves in elderly patients with conditions such as complicated cardiac reoperation, low left ventricular function, respiratory dysfunction, liver dysfunction, and severe frailty. Conversely, we believe that mechanical valves are desirable in patients with insulin-dependent diabetes mellitus, oral administration of certain drugs such as steroids, chemotherapy, and uncontrolled parathyroid function.

In conclusion, this RD-AV system has shown excellent results in terms of hemodynamic performance, durability, and safety. It may be beneficial in complex combined procedures in patients on hemodialysis with prior CABG.

\section{REFERENCES}

Ando T, Briasoulis A, Holmes AA, et al. 2016. Transcatheter aortic valve replacement versus surgical aortic valve replacement in patients with previous coronary artery bypass surgery: A systematic review and metaanalysis. Int J Cardiol 215:14-19.

Andreas M, Coti I, Rosenhek R, et al. 2019. Intermediate-term outcome of 500 consecutive rapid-deployment surgical aortic valve procedures. Eur J Cardiothorac Surg 55:527-33.
Baumgartner H, Falk V, Bax JJ, et al. 2017. 2017 ESC/EACTS Guidelines for the management of valvular heart disease. Eur Heart J 38:2739-91.

Berretta P, Arzt S, Fiore A, et al. 2020. Trends of sutureless and rapid deployment valves: An 11-year experience from the Sutureless and Rapid Deployment International Registry. Eur J Cardiothorac Surg 58:1054-62.

Chan PG, Sultan I, Gleason TG, Navid F, Kilic A. 2019. Mechanical versus bioprosthetic valves in patients on dialysis. J Thorac Dis 11:1996-2005.

Conte JV, Gleason TG, Resar JR, et al. 2016. Transcatheter or surgical aortic valve replacement in patients with prior coronary artery bypass grafting. Ann Thorac Surg 101:72-9.

Coti I, Haberl T, Scherzer S, et al. 2020. Outcome of rapid deployment aortic valves: Long-term experience after 700 implants. Ann Cardiothorac Surg 9:314-21.

Greason KL, Mathew V, Suri RM, et al. 2014. Transcatheter versus surgical aortic valve replacement in patients with prior coronary artery bypass graft operation: A PARTNER trial subgroup analysis. Ann Thorac Surg 98:1-7.

Jegaden O, Lapeze J, Farhart F, de Gavigney G. 2012. Aortic valve stenosis after previous coronary bypass: Transcatheter valve implantation or aortic valve replacement? J Cardiothorac Surg 7:47-9.

Kocher AA, Laufer G, Haverich A, et al. 2013. One-year outcomes of the Surgical Treatment of Aortic Stenosis With a Next Generation Surgical Aortic Valve (TRITON) trial: A prospective multicenter study of rapiddeployment aortic valve replacement with the EDWARDS INTUITY Valve System. J Thorac Cardiovasc Surg 145:110-16.

Nakatsu T, Minakata K, Tanaka S, Minatoya K. 2019. PROGRESSKyoto Investigators. Intermediate-term outcomes of aortic valve replacement with bioprosthetic or mechanical valves in patients on hemodialysis. J Thorac Cardiovasc Surg 157:2177-86. 